\title{
Charges and Energy in Chern-Simons Theories and Lovelock Gravity
}

\author{
G. Allemandi; M.Francaviglia ${ }^{\dagger}$ M. Raiteri ${ }^{\ddagger}$ \\ Dipartimento di Matematica, Università degli Studi di Torino, \\ Via Carlo Alberto 10, 10123 Torino, Italy
}

\begin{abstract}
Starting from the $S O(2,2 n)$ Chern-Simons form in $(2 n+1)$ dimensions we calculate the variation of conserved quantities in Lovelock gravity and Lovelock-Maxwell gravity through the covariant formalism developed in [23]. Despite the technical complexity of the Lovelock Lagrangian we obtain a remarkably simple expression for the variation of the charges ensuing from the diffeomorphism covariance of the theory. The viability of the result is tested in specific applications and the formal expression for the entropy of Lovelock black holes is recovered.
\end{abstract}

PACS numbers: 04.50.+h; 04.20.Fy; 04.70.Bw

\section{Introduction}

Lovelock gravity can be considered as the most general extension of General Relativity to higher dimensions: in fact, even if it contains higher curvature interaction terms, it is nevertheless governed by field equations which are just of the second order in the metric field [39, 40]. Far from being just a mathematical curiosity, Lovelock gravity as well as higher curvature theories in general, have received in the recent past a renewed interest motivated by the hope of learning something about the nature of quantum gravity. As a matter of facts, higher curvature interaction terms arise in the study of back-reactions of quantum field energy as well as in the low-energy limit of string theory (see [9, 11, 30, 42]).

In this paper we address the problem to calculate the charges associated to the diffeomorphism symmetries of Lovelock gravity in any odd dimensional spacetime. To this end we make use of the recipe worked out in [23] to algorithmically define (the variation of) conserved quantities directly from the equations of motion. One of the major advantages of the formalism developed

\footnotetext{
*allemandi@dm.unito.it

†francaviglia@dm.unito.it

‡raiteri@dm.unito.it
} 
in [23] is that it enables to establish the transformation rules acting on conserved quantities in the transition from different, but nevertheless on-shell equivalent, Lagrangian theories. This property is well suited to tackle with our problem. It was indeed shown in $[6,13]$ that a special case of Lovelock gravity (with a fixed choice of the Lagrangian coefficients) in $D=2 n+1$ dimensions can be derived from a Chern-Simon theory of the group $S O(2,2 n)$ (or $S O(1,2 n+1)$ ). Combining together this remarkable result with the formalism of [23] for conserved quantities, we shall show that the calculation of conserved quantities for Lovelock gravity becomes rather easier if we first compute them in the Chern-Simons framework and we take then into account the suitable transformation rules on the field variables as well as on the infinitesimal generators of symmetries. Even though we end up in this way with an expression which holds true only for a very special case of Lovelock gravity, the contribution to conserved quantities coming from each higher curvature interaction term can be nevertheless isolated. This property allows one to derive a general formula for (the variation of) conserved quantities for any Lovelock theory, i.e. with completely arbitrary choice of the coefficients in front of each curvature interaction term.

Let us now analyse the problem in detail. The $D=2 n+1$ dimensional ChernSimons Lagrangian for the anti-de Sitter gauge group $S O(2,2 n)$ is written in terms of a gauge connection

$$
A=A_{\mu}^{A B} d x^{\mu} J_{A B}
$$

where $\mu=0, \ldots, 2 n ; A, B=0, \ldots, 2 n+1$; and $J_{A B}$ are the Lie algebra generators:

$$
\left[J_{A B}, J_{C D}\right]=\frac{1}{2}\left\{-J_{A C} \eta_{B D}+J_{A D} \eta_{B C}+J_{B C} \eta_{A D}-J_{B D} \eta_{A C}\right\}
$$

with $\eta_{A B}=(-1,1, \ldots, 1,-1)$.

The Lagrangian turns out to be [13]: ${ }^{1}$

$$
L_{1}\left(j^{1} A\right)=\kappa \int_{0}^{1} d t<A \wedge\left(t d A+t^{2} A^{2}\right)^{n}>
$$

where $<>$ denotes the invariant form:

$$
<J_{A_{1} B_{1}} \ldots J_{A_{n+1} B_{n+1}}>=\epsilon_{A_{1} B_{1} \ldots A_{n+1} B_{n+1}}
$$

According to [6] the constant $k$ in (3) is fixed as:

$$
\kappa=\frac{l}{(D-2) ! \Omega_{D-2}}, \quad l=\text { const }
$$

where $\Omega_{D-2}$ is the area of the $(D-2)$-dimensional unit sphere and $l$ has the dimension of a length. The Euler-Lagrange field equations ensuing from (3) turn out to be:

$$
\frac{\delta L_{1}}{\delta A^{A_{n+1} B_{n+1}}}=\kappa \epsilon_{A_{1} B_{1} \ldots A_{n+1} B_{n+1}} F^{A_{1} B_{1}} \wedge \ldots \wedge F^{A_{n} B_{n}}=0
$$

\footnotetext{
${ }^{1}$ In the sequel we shall make use of the notation $f\left(j^{k} y\right)$ to denote a quantity " $f$ " depending on the variables $y$ together with their derivatives up to the order $k$.
} 
where $F^{A B}=d A^{A B}+A^{A}{ }_{C} \wedge A^{C B}$ is the field strength of the gauge connection. Furthermore, the set of generators $J_{A B}$ can be split as $J_{A B}=\left(J_{a b}, P_{a}\right)$, $a=0, \ldots, D-1$; namely: into the generators $J_{a b}$ of Lorentz rotations in $D$ dimensions and the generators $P_{a}:=J_{a, 2 n+1}$ of inner translations (see for example [1], [13], [51]). Accordingly, the $S O(2,2 n)$ gauge connection can be decomposed as follows:

$$
A=\omega^{a b} J_{a b}+\frac{1}{l} e^{a} P_{a}
$$

This assumption implies that the field strength in turn decomposes as:

$$
F=\left(R^{a b}+\frac{1}{l^{2}} e^{a} \wedge e^{b}\right) J_{a b}+\frac{1}{l} T^{a} P_{a}
$$

where

$$
R^{a b}=d \omega^{a b}+\omega^{a}{ }_{c} \wedge \omega^{c b}
$$

is the field strength of the $S O(1,2 n)$ gauge connection $\omega^{a b}$, while

$$
T^{a}=d e^{a}+\omega^{a}{ }_{b} \wedge e^{b}
$$

is the torsion $2-$ form.

Substituting expressions (7) and (8) into (3) and taking into account that the only terms which do not vanish are those of the kind $<J_{a_{1} b_{1}} \ldots J_{a_{n} b_{n}} P_{a}>=$ $\epsilon_{a_{1} b_{1} \ldots a_{n} b_{n} a}$ we can rewrite the Chern-Simons Lagrangian in terms of the new dynamical fields $(\omega, e)$. Apart from boundary terms we obtain:

$$
L_{2}\left(e, j^{1} \omega\right)=\kappa \sum_{p=0}^{n} c_{p} \mathcal{L}_{p}\left(e, j^{1} \omega\right)
$$

where

$$
\begin{aligned}
& c_{p}=\frac{1}{(D-2 p)}\left(\begin{array}{l}
n \\
p
\end{array}\right) \frac{1}{l^{D-2 p}} \\
& \mathcal{L}_{p}\left(e, j^{1} \omega\right)=\epsilon_{a_{1} \ldots a_{D}} R^{a_{1} a_{2}} \wedge \ldots \wedge R^{a_{2 p-1} a_{2 p}} \wedge e^{a_{2 p+1}} \wedge \ldots \wedge e^{a_{D}}
\end{aligned}
$$

The Lagrangian (11) turns out to be the sum of dimensionally continued Euler densities [6, 13, 47] and we shall refer to it as the Euler-Chern-Simons Lagrangian. Notice that in the expressions (13) the "vielbein" $e^{a}$ appears without derivatives and the gauge connection $\omega^{a b}$ enters only through its field strength $R$. This remarkable property is due to the fact that all the terms involving explicitly the torsion and/or the gauge connection $\omega$ are pushed, through integrations by parts, into boundary terms which are discarded in the expression (11). Notice also that, until field equations are not yet solved, no a priori rule exists relating the fields $e^{a}$ and $\omega^{a b}$ : they are completely independent. ${ }^{2}$

\footnotetext{
${ }^{2}$ Geometrically speaking we are assuming that the Lagrangian $L_{2}$ is based on a configuration bundle $Y$ which is a product bundle $Y=Y_{1} \times_{M} Y_{2}$ with fibered coordinates $\left(x^{\mu}, e_{\nu}^{a}, \omega_{\rho}^{b c}\right)$, where $\left(x^{\mu}, e_{\nu}^{a}\right)$ and $\left(x^{\mu}, \omega_{\rho}^{b c}\right)$ are fibered coordinates on $Y_{1}$ and $Y_{2}$, respectively. The configuration bundle $Y$ is thence a gauge natural bundle admitting a principal $S O(1,2 n)$ bundle $Q$ as structure bundle $[18,38]$. Accordingly $Y_{1}$ is the spin-frame bundle [19] while $Y_{2}=J^{1} Q / S O(1,2 n)$ is the connection bundle; see $[38,45]$
} 
Varying the Lagrangian (11) with respect to the independent fields $(e, \omega)$ we obtain, respectively, the field equations:

$$
\left\{\begin{array}{l}
\frac{\delta L_{2}}{\delta e^{a}}=\frac{\kappa}{l} \epsilon_{a a_{1} b_{1} \ldots a_{n} b_{n}} \hat{R}^{a_{1} b_{1}} \wedge \ldots \wedge \hat{R}^{a_{n} b_{n}}=0 \\
\frac{\delta L_{2}}{\delta \omega^{\omega_{n} b_{n}}}=\frac{n \kappa}{l} \epsilon_{a a_{1} b_{1} \ldots a_{n} b_{n}} T^{a} \wedge \hat{R}^{a_{1} b_{1}} \wedge \ldots \wedge \hat{R}^{a_{n-1} b_{n-1}}=0
\end{array}\right.
$$

where

$$
\hat{R}^{a b}=R^{a b}+\frac{1}{l^{2}} e^{a} \wedge e^{b}
$$

Notice that field equations (14) are dynamically equivalent to field equations (6) once the substitution (7) is taken into account. Namely, the difference between the two sets of equations is just a matter of notation.

If we now consider the particular solution $T^{a}=0$ of the second set of field equations (14), it turns out that the $S O(1,2 n)$ connection $\omega^{a b}$ is the spin connection of the vielbein $e^{a}$ :

$$
\omega_{b \mu}^{a}=e_{\alpha}^{a}\left(\Sigma_{\beta \mu}^{\alpha}-\Sigma_{\beta}{ }^{\alpha}{ }_{\mu}+\Sigma_{\mu \beta}{ }^{\alpha}\right) e_{b}^{\beta}, \quad \Sigma_{\beta \mu}^{\alpha}=e_{c}^{\alpha} \partial_{[\beta} e_{\mu]}^{c}
$$

and

$$
R^{a b}{ }_{\mu \nu}=e_{\rho}^{a} e_{\sigma}^{b} R_{\mu \nu}^{\rho \sigma}\left(j^{2} g\right)
$$

where $R^{\rho \sigma}{ }_{\mu \nu}\left(j^{2} g\right)$ is the Riemann tensor of the metric $g_{\mu \nu}=\eta_{a b} e_{\mu}^{a} e_{\nu}^{b}$. Inserting (17) back into the Lagrangian (11) we end up with a special Lovelock Lagrangian, namely:

$$
L_{3}\left(j^{2} g\right):=\left.L_{2}\left(e, j^{1} \omega\right)\right|_{T^{a}=0}=\kappa \sum_{p=0}^{n} \bar{c}_{p} \overline{\mathcal{L}}_{p}\left(j^{2} g\right)
$$

where:

$$
\begin{aligned}
& \bar{c}_{p}=\left(\begin{array}{l}
n \\
p
\end{array}\right) \frac{(2 n-2 p) !}{l^{D-2 p}} \\
& \overline{\mathcal{L}}_{p}\left(j^{2} g\right)=\frac{1}{2^{p}} \sqrt{g} \delta_{\nu_{1} \ldots \nu_{2 p}}^{\mu_{1} \ldots \mu_{2 p}} R_{\mu_{1} \mu_{2}}^{\nu_{1} \nu_{2}} \ldots R_{\mu_{2 p-1} \nu_{2 p}}^{\nu_{2 p-1} \nu_{2 p}}
\end{aligned}
$$

and $\delta_{\nu_{1} \ldots \nu_{2 p}}^{\mu_{1} \ldots \mu_{2 p}}$ denotes the totally skew-symmetric product of Kronecker deltas normalized to have values $0, \pm 1 .^{3}$ In particular: $\overline{\mathcal{L}}_{0}=\sqrt{g}$ gives rise to the term corresponding to a cosmological constant $\Lambda$ proportional to $-1 / l^{2}$ (or proportional to $1 / l^{2}$ if we had started with the group $S O(1,2 n+1)$ ); $\overline{\mathcal{L}}_{1}=\sqrt{g} R$ is the Hilbert Lagrangian in dimension $2 n+1$ and $\overline{\mathcal{L}}_{2}=\sqrt{g}\left(R^{2}-4 R_{\beta}^{\alpha} R_{\alpha}^{\beta}+R_{\alpha \beta}^{\mu \nu} R_{\mu \nu}^{\alpha \beta}\right)$ is the Gauss-Bonnet term. Notice that the coefficients in the Lovelock Lagrangian (18) are uniquely fixed and in fact dictated by the initial Chern-Simons form

\footnotetext{
${ }^{3}$ In writing (20) we made use of the fact that, according to our notation,$$
\epsilon^{\mu_{1} \ldots \mu_{2 p} \alpha_{2 p+1} \ldots \alpha_{D}} \epsilon_{\nu_{1} \ldots \nu_{2 p} \alpha_{2 p+1} \ldots \alpha_{D}}=(D-2 p) ! \delta_{\nu_{1} \ldots \nu_{2 p}}^{\mu_{1} \ldots \mu_{2 p}}
$$ 
(3); see $[6,13,15]$. From now on we shall refer to the Lagrangian (18) as Lovelock-Chern-Simons Lagrangian.

Let us now summarize. We have introduced so far three different Lagrangians; see expressions (3), (11) and (18). The transition among them can be schematically drawn as follows:

$$
L_{1}\left(j^{1} A\right) \stackrel{A=A(\omega, e)}{\Longrightarrow} L_{2}\left(e, j^{1} \omega\right) \stackrel{T=0}{\Longrightarrow} L_{3}\left(j^{2} g\right)
$$

Notice however that the equivalence between the first two Lagrangians, as already explained, holds true off-shell. On the contrary, the equivalence between $L_{2}$ and $L_{3}$ is just an on-shell equivalence since it holds true only along the space of solutions with $T=0$.

The goal of the present paper is to calculate the conserved quantities, in particular the energy, for each one of the Lagrangians in (22). We point out that this task is more subtle than it could first look like. The reason is mainly due to two different causes.

1. Let us suppose that we are somehow able to calculate the charge $Q\left(L_{1}, \Xi\right)$ associated to the Lagrangian $L_{1}\left(j^{1} A\right)$ and relative to an infinitesimal generator of symmetries $\Xi$ which is common to both the Lagrangians $L_{1}$ and $L_{2}$. According to the prescription (22) we could be induced to identify the charge $Q\left(L_{2}, \Xi\right)$, relative to the second Lagrangian in (22) and associated with the same generator $\Xi$, with the expression $Q\left(L_{2}, \Xi\right)=$ $\left.Q\left(L_{1}, \Xi\right)\right|_{A(\omega, e)}$, i.e. by merely inserting the splitting (7) into $Q\left(L_{1}, \Xi\right)$. This rule could fail in giving the right expression. Indeed we stress that in the transition from the Lagrangian (3) to the Lagrangian (11) divergence terms have to be discarded. As far as field equations are concerned, those divergence terms are clearly irrelevant. On the contrary, they can become strongly relevant when dealing with conserved quantities. For example, conserved quantities calculated via Noether theorem, Hamiltonian or Hamilton-Jacobi-like methods are all sensitive to divergence terms of the Lagrangian (see, e. g., [10, 12, 24, 32, 33, 44]).

2. Let us now suppose we have been somehow able to bypass the aforementioned problem. There remain still another open question. Indeed, conserved quantities of a field theory are mathematical expressions which are attributed the meaning of physical observables. They are built out by keeping into account the symmetry properties of the field theory we are dealing with. Hence, we have to know the relationships interplaying among the symmetries of the three Lagrangians (22) (or their field equations) if we want to establish a correspondence among their charges, too. Entering into the details of the matter, we observe that the Chern-Simons theory is a gauge natural theory; see $[18,19,20,27,38]$. It is based on a configuration bundle $\mathcal{C}$ which is the bundle of connections $\mathcal{C}=J^{1} P / S O(2,2 n)$, where $P$ denotes a principal bundle with Lie group $S O(2,2 n)$, called the structure bundle of the theory; see [45]. Fibered coordinates on $\mathcal{C}$ are 
$\left(x^{\mu}, A_{\nu}^{A B}\right)$. The most general infinitesimal generator of symmetries for the equations of motion (6) is a projectable vector field $\Xi_{P}$ on $P$ of the kind

$$
\Xi_{P}=\xi^{\mu} \partial_{\mu}+\Xi^{A B} \rho_{A B}
$$

where $\rho_{A B}$ is a local basis of right invariant vector fields on $P$ (in a trivialization $(x, g)$ of $P$ we have $\rho=(g \partial / \partial g), g \in S O(2,2 n))$. The vector field $\Xi_{P}$ induces a transformation of the dynamical fields according to the rule:

$$
£_{\Xi_{P}} A_{\mu}^{A B}=\xi^{\rho} d_{\rho} A_{\mu}^{A B}+d_{\mu} \xi^{\rho} A_{\rho}^{A B}+\stackrel{A}{D} \Xi_{\mu} \Xi^{A B}
$$

and pure gauge transformations correspond to $\xi^{\mu}=0$. Therefore the charge $Q\left(L_{1}, \Xi_{P}\right)$ associated to this symmetry must depend, in general, on the dynamical field $A$, on the coefficients $\xi^{\mu}$ and $\Xi^{A B}$ together with their derivatives up to a fixed (finite) order. If we now consider the splitting

$$
\Xi^{A B}=\left(\Xi^{a b}, \Xi^{a}\right)
$$

of the vertical part of the vector field $\Xi_{P}$ into "rotational" part and "translational" part, we obtain from (7) and (24)

$$
\begin{aligned}
£_{\Xi_{P}} \omega_{\mu}^{a b} & =\xi^{\rho} d_{\rho} \omega_{\mu}^{a b}+d_{\mu} \xi^{\rho} \omega_{\rho}^{a b}+\stackrel{\omega}{D} \mu \Xi^{a b}+\frac{1}{l} e_{\mu}^{a} \Xi^{b}-\frac{1}{l} e_{\mu}^{b} \Xi^{a} \\
£_{\Xi_{P}} e_{\mu}^{a} & =\xi^{\rho} d_{\rho} e_{\mu}^{a}+d_{\mu} \xi^{\rho} e_{\rho}^{a}+l \stackrel{\omega}{D_{\mu}} \Xi^{a}-\Xi^{a}{ }_{c} e_{\mu}^{c}
\end{aligned}
$$

and, if we do not assume a priori the condition $T^{a}=0$, field equations (14) are separately invariant for the transformation (26) only when we set $\Xi^{a}=0 .^{4}$

It follows that the most general infinitesimal generator of symmetries for each one of the equations of motion (14) is a projectable vector field $\Xi_{Q}$ on the relevant $S O(2 n, 1)$ principal bundle $Q$ of the theory (see footnote 2 ) which, in turn, is a subbundle of $P$. In coordinates:

$$
\Xi_{Q}=\xi^{\mu} \partial_{\mu}+\Xi^{a b} \rho_{a b}
$$

where now $\rho_{a b}$ is a local basis of right invariant vector fields on $Q$. The charge $Q\left(L_{2}, \Xi_{Q}\right)$ associated to the vector field (27) must depend, in general, on the dynamical fields $(e, \omega)$, on the coefficients $\xi^{\mu}$ and $\Xi^{a b}$ together with their derivatives up to a fixed order and can be suitable obtained from $Q\left(L_{1}, \Xi_{P}\right)$ through the prescription (7) on the dynamical variables and the prescription $\Xi^{a}=0$ on the generators of symmetries.

\footnotetext{
${ }^{4}$ Basically, the Chern-Simons symmetry is broken when we declare that the dynamical fields $(e, \omega)$ are independent fields or, in other words, when we assume that the configuration bundle $Y=Y_{1} \times_{M} Y_{2}$ has a product bundle structure. From (26) it is clear that "inner translations" of the AdS group, namely those generated by the components $\Xi^{a}$, induce transformations on the configuration bundle which do not respect the product bundle structure.
} 
Let us finally draw our attention to the Lovelock-Chern-Simons Lagrangian (18). The latter Lagrangian is a natural (also said covariant or diffeomorphism invariant; see $[25,33,38]$ ) Lagrangian and the generators of symmetries are (not necessarily Killing) vector fields $\xi=\xi^{\mu} \partial_{\mu}$ on the base manifold (i.e. the $D$-dimensional spacetime). It follows that the charges $Q\left(L_{3}, \xi\right)$ must depend, in general, on the dynamical field $g$ and on the coefficients $\xi^{\mu}$ together with their derivatives up to a fixed order. To obtain a correspondence between $Q\left(L_{2}, \Xi_{Q}\right)$ and $Q\left(L_{3}, \xi\right)$ the prescriptions (16) and (17) alone are not sufficient since we also need a prescription to fix the coefficients $\Xi^{a b}=\Phi^{a b}\left(\xi^{\mu}, d_{\nu} \xi^{\mu}, \ldots\right)$ in terms of the coefficients $\xi^{\mu}$ and their derivatives up to a fixed order, namely we need to select a lift of the vector field $\xi$ up to the structure bundle $Q$ of the Lagrangian $L_{2}$. Notice that the rule $\Xi^{a b}=0$ is only local and it is not preserved (in general) by the automorphisms of the structure bundle $Q$, meaning that we have to resort to some other covariant lift. If we are able to select a lift $\Xi^{a b}=\Phi^{a b}\left(\xi^{\mu}, d_{\nu} \xi^{\mu}, \ldots\right)$ which is mathematically well-defined (i.e. a covariant lift) as well as physically viable (i.e. it allows to reproduce the expected physical values for conserved quantities in specific applications) we have the following scheme:

$$
Q\left(L_{1}\left(j^{1} A\right), \Xi_{P}\right) \underset{\Xi^{a}=0}{\stackrel{A=A(\omega, e)}{\longrightarrow}} Q\left(L_{2}\left(e, j^{1} \omega\right), \Xi_{Q}\right) \underset{\Xi^{a b}=\Phi^{a b}(\xi)}{\stackrel{T=0}{=}} Q\left(L_{3}(g), \xi\right)
$$

governing the transition among conserved quantities.

In the sequel we shall follow a recipe to calculate conserved quantities which has been recently worked out in [23]. The formalism developed in [23] is in fact well suited to tackle the issue we are dealing with, mainly because it allows to overcome both the problems we have just outlined. Basically, the construction of conserved quantities is defined as follows. Given a field theory the dynamics of which is derived from a Lagrangian $L$ and given a vector field $\Xi$ which is an infinitesimal generator of symmetries for the equations of motions $\frac{\delta L}{\delta y}$, one can construct the variational Lagrangian $L^{\prime}(L, \Xi)$ through the contraction of field equations with the Lie derivatives of fields, i.e. $L^{\prime}(L, \Xi)=-\frac{\delta L}{\delta y^{2}} £ \Xi y^{i}$. Handling the variational Lagrangian with the tools of Variational Calculus in jet bundles it is possible to algorithmically define the variation $\delta Q\left(L^{\prime}, \Xi\right)$ of covariantly conserved quantities along a one-parameter family of solutions.

Since the variation of conserved quantities is obtained directly from the equations of motion the drawback related to problem 1) above is automatically overcome: the procedure is not affected by whichever divergence term we add to a given Lagrangian since field equations are insensitive to it. Moreover, also the problem raised in problem 2) can be easily bypassed. Indeed, by imposing the equivalence between the variational Lagrangian $L^{\prime}\left(L_{2}, \Xi_{Q}\right)$ (built out starting from the equations of motion (14) and the generator of symmetries (27)) and the variational Lagrangian $L^{\prime}\left(L_{3}, \xi\right)$ (where $\xi$ is any spacetime vector field) a preferred lift $\Xi_{Q}=\Phi\left(\xi^{\mu}, d_{\nu} \xi^{\mu}, \ldots\right)$ of spacetime vector fields is automatically 
ruled out from the theory and it allows to complete the diagram (28). The selected lift turns out to be the so-called generalised Kosmann lift; see [19, 27, 28].

Once we have understood the rules which allow us to make the transition from the (variation of the) conserved quantities $\delta Q\left(L_{1}^{\prime}, \Xi_{P}\right)$ ensuing from the Chern-Simons Lagrangian (3) and the (variation of the) conserved quantities $\delta Q\left(L_{3}^{\prime}, \xi\right)$ associated to the Lagrangian (18), the latter quantities can be easily computed from the former ones. Moreover, even if the quantities obtained in this way are relative to the whole Lagrangian (18), the contribution coming from each Lagrangian $\overline{\mathcal{L}}_{p}$ can be easily recognized and, therefore, we can define the conserved quantities for a generic Lovelock Lagrangian with arbitrary coefficients in front of each term. Hence, despite the technical complexity of the Lovelock Lagrangian, we obtain a remarkably simple expression for its charges.

The rest of the paper is organized as follows. In section 2 we shall shortly summarize the formalism developed in [23] to obtain the variation of conserved quantities directly from the equations of motion. We then apply it to calculate conserved quantities relative to the Chern-Simons form (3). Following the recipe schematically drawn in (28) we are then able to define the variation of conserved quantities for Lovelock gravity. A straightforward generalization of the aforementioned formalism will allow the generalization to Lovelock-Maxwell gravity. In section 3 the viability of the results previously obtained is tested for a five-dimensional charged black hole solution and the first law of black hole mechanics directly follows from the homological properties conserved quantities must satisfy. In section 4 the formal expression for the entropy of Lovelock stationary black holes is finally calculated and it agrees with previous definitions; see $[33,35,49]$.

\section{Conserved Quantities}

In order to make the paper self-contained we shall here summarize the formalism to obtain the variation of conserved quantities directly from the equations of motion. Since we are interested only in the application of the technique to the Chern-Simons theory and to Lovelock gravity, we shall skip the rigorous geometric framework and we refer the reader to [23] for a deeper insight into the mathematical details of the matter. To stimulate the interest of physicallyoriented readers we shall keep as much as possible the formal setting and the technical details to a minimum.

Let us consider a field theory described through a Lagrangian of order $k$. It can be locally written as $L=\mathcal{L}\left(y^{i}, \ldots, y_{\mu_{1} \ldots \mu_{k}}^{i}\right) d s$ where $\mathcal{L}$ is the Lagrangian density, $d s=d x^{1} \wedge \ldots \wedge d x^{D}$ is the local volume element of the $D$-dimensional spacetime and we have collectively denoted with $y^{i}$ all the dynamical fields.

The variation $\delta_{X} \mathcal{L}$ of the Lagrangian density with respect to a vector field 
$X=X^{i} \frac{\partial}{\partial y^{i}}=\delta y^{i} \frac{\partial}{\partial y^{i}}$ can be generally written, through a well known integration by parts procedure, as follows:

$$
\delta_{X} \mathcal{L}\left(j^{k} y\right)=e_{i}\left(j^{2 k} y\right) X^{i}+d_{\lambda} F^{\lambda}\left(j^{2 k-1} y, j^{k-1} X\right)
$$

or, in terms of differential forms, as

$$
\delta_{X} L=e(L, X)+d F(L, X)
$$

Here the $D$-form

$$
e(L, X)=e_{i}\left(j^{2 k} y\right) X^{i} d s
$$

and the $(D-1)-$ form

$$
\left.F(L, X)=F^{\lambda}\left(j^{2 k-1} y, j^{k-1} X\right) d s_{\lambda}, \quad d s_{\lambda}=i_{\lambda}\right\rfloor d s
$$

are called, respectively, the Euler-Lagrange form and the Poincarè-Cartan form. Obviously field solutions are those field configurations $y^{i}=\sigma^{i}(x)$ which satisfy the Euler-Lagrange equations:

$$
\left.e_{i}\left(j^{2 k} y\right)\right|_{y=\sigma(x)}=0
$$

Let us now suppose that $\Xi$ is an infinitesimal generator of symmetries, namely a projectable vector field $\Xi=\xi^{\mu}(x) \frac{\partial}{\partial x^{\mu}}+\Xi^{i}(x, y) \frac{\partial}{\partial y^{i}}$ which leaves field equations invariant:

$$
\delta_{\Xi} e_{i}\left(j^{2 k} y\right)=0
$$

The contraction of the field equations (33) with the Lie derivatives $£ \Xi y^{i}$ of the field originates, apart for a sign, the so-called variational Lagrangian

$$
L^{\prime}(L, \Xi):=-e_{i}\left(j^{2 k} y\right) £ \Xi y^{i} d s
$$

which depends on the dynamical fields $y^{i}$ and the components $\xi^{\mu}$ and $\Xi^{i}$ of the vector field $\Xi$; it is clearly vanishing along solutions. According to the first variation formula (30), the variation of the variational Lagrangian $L^{\prime}$ yields: ${ }^{5}$

$$
\delta_{X} L^{\prime}=e\left(L^{\prime}, X\right)+d F\left(L^{\prime}, X\right)
$$

Moreover, since we have assumed that $\Xi$ is an infinitesimal generator of symmetries it turns out that the variational Lagrangian is a pure divergence and therefore it is variationally trivial; see [23]. This means that $e\left(L^{\prime}, X\right)=0$ and, consequently, (36) reduces to the conservation law

$$
d F\left(L^{\prime}, X\right)=\delta_{X} L^{\prime} \simeq 0
$$

where $\simeq$ denotes equality on-shell. Moreover the Poincarè-Cartan morphism $F\left(L^{\prime}, X\right)$ in $(37)$ is linear in the Lie derivatives $£_{\Xi} y$ and their derivatives up to

\footnotetext{
${ }^{5}$ We remark that, while it is not restrictive to set $\delta \xi^{\mu}=0$ as the components $\xi^{\mu}$ do not depend on the dynamical fields, we shall instead allow $\delta \Xi^{i} \neq 0$.
} 
a fixed order. Since in all physically relevant theories (e.g. in all gauge natural theories; see $[18,20,38])$ the Lie derivatives of the fields with respect to a vector field can be written as linear combinations in the components of the vector field itself together with their (covariant) derivatives, it turns out that also the Poincarè-Cartan morphism $F\left(L^{\prime}, X\right)$ is a linear combination of the coefficients $\xi^{\mu}$ and $\Xi^{i}$ of the vector field $\Xi$ together with their derivatives up to a finite order which depends on the particular theory we are dealing with. This allows us to implement the so-called Spencer cohomology [18, 20, 25, 29]. Namely, the Poincarè-Cartan morphism $F\left(L^{\prime}, X\right)$, through repeated covariant integrations by parts with respect to the components of $\Xi$, can be alghoritmically rewritten as [23]:

$$
F\left(L^{\prime}, X\right)=\tilde{F}\left(L^{\prime}, X\right)+d \mathcal{U}\left(L^{\prime}, X\right) \simeq d \mathcal{U}\left(L^{\prime}, X\right)
$$

where the term $\tilde{F}\left(L^{\prime}, X\right)$ turns out to be proportional to the field equations together with their derivatives and is thence vanishing on-shell, while the $(D-2)$ form $\mathcal{U}\left(L^{\prime}, X\right)$ is the (covariant) potential. The potential depends in turn on the dynamical fields $y^{i}$, on the components $\left(\xi^{\mu}, \Xi^{i}\right)$ of the infinitesimal generator of symmetries and on the components of the variational field $X$ together with their derivatives up to a suitable order. Given a field configuration $y=\sigma(x)$ and a $(D-1)$-dimensional region $\Sigma$ of spacetime with boundary $\partial \Sigma$, the integral

$$
\begin{aligned}
\delta_{X} Q_{\Sigma}(\sigma, \Xi):=\int_{\Sigma} F\left(L^{\prime}, X\right) & =\int_{\Sigma} \tilde{F}\left(L^{\prime}, X\right)+\int_{\partial \Sigma} \mathcal{U}\left(L^{\prime}, X\right) \\
& \simeq \int_{\partial \Sigma} \mathcal{U}\left(L^{\prime}, X\right)
\end{aligned}
$$

is assumed to be the variation $\delta_{X} Q$ of the charge $Q$ along a one-parameter family of field configuration described by $X$ and relative to the symmetry originated by $\Xi$. If $y=\sigma(x)$ is a solution of field equations and $X$ is a solution of the linearized field equations (i.e. $X$ is tangent to the space of solutions) the quantity $\delta_{X} Q_{\Sigma}(\sigma, \Xi) \simeq \int_{\partial \Sigma} \mathcal{U}\left(L^{\prime}, X\right)$ describes the variation of the charge $Q$ moving from $\sigma$ to nearby solutions along a path in the space of solutions determined by $X$. Moreover if the region $\Sigma$ is a (portion of) a Cauchy hypersurface with a boundary $\partial \Sigma$ and, in addition, the spacetime projection $\xi$ of the vector field $\Xi$ is transverse to $\Sigma$, we agree to identify $\delta_{X} Q_{\Sigma}$ with the variation of the energy $E(\partial \Sigma, \xi, X)$ enclosed inside $\partial \Sigma$. We stress that, once we fix $\partial \Sigma$, there exist many "energies", depending both on the symmetry vector field $\xi$ and on the variational vector field $X ;[2,12,26,37]$. The former vector determines, via its flow parameter, how the hypersurface $\Sigma$ evolves as the "time" flows. The vector field $X$ fixes instead the boundary conditions on the dynamical fields (e.g. Dirichlet or Newmann boundary conditions). Each choice of the pair $(\xi, X)$ gives rise to a particular realization of a physical system endowed thence with its own "energy content".

Summarizing, we obtained the variation of conserved quantities starting from the equations of motion through a two-step procedure. The first step is nothing but an integration by parts of the variational Lagrangian with respect to the 
vector field $X$ (see (36)). The second step amounts to perform a second integration by parts of the Poincarè-Cartan morphism $F\left(L^{\prime}, X\right)$ with respect to the vector field $\Xi$ (see (38)). We remark that, a priori, there exists no guarantee that the resulting quantity $\delta_{X} Q_{\Sigma}$ is in fact the variation of a functional $Q$ of the intrinsic geometry of the boundary $\partial \Sigma$. This becomes true only if appropriate boundary conditions are chosen; see [4, 8, 10, 12, 14, 26, 32, 37, 44].

The viability of such a procedure has been tested in [23] in the realm of natural and gauge natural theories of gravitations. The physically expected values (or better, the commonly accepted expressions) for conserved quantities, in particular for the energy, have been there reproduced. ${ }^{6}$ Encouraged by those positive results we aim now to apply the formalism to Chern-Simons and Lovelock theories.

The variational Lagrangian $L^{\prime}\left(L_{1}, \Xi\right)$ for the Chern-Simons theory is easily obtained from the equations of motion (6) :

$$
L^{\prime}\left(L_{1}, \Xi\right)=-\kappa \epsilon_{A_{1} B_{1} \ldots A_{n+1} B_{n+1}} F^{A_{1} B_{1}} \wedge \ldots \wedge F^{A_{n} B_{n}} £ \Xi A^{A_{n+1} B_{n+1}}
$$

Taking into account that the Lie derivative (24) can be conveniently rewritten in a manifestly covariant form as:

$$
£_{\Xi} A_{\lambda}^{A B}=\xi^{\rho} F_{\rho \lambda}^{A B}+\stackrel{(A)}{D}_{\lambda} \Xi_{(V)}^{A B} \quad\left(\Xi_{(V)}^{A B}=\Xi^{A B}+A_{\sigma}^{A B} \xi^{\sigma}\right)
$$

the variation of (41) turns out to be (first step):

$$
\delta_{X} L^{\prime}\left(L_{1}, \Xi\right)=d_{\alpha} \mathcal{F}^{\alpha}\left(L^{\prime}\left(L_{1}, \Xi\right), X\right)
$$

where

$$
\begin{aligned}
\mathcal{F}^{\alpha}\left(L^{\prime}\left(L_{1}, \Xi\right), X\right)= & -\frac{\kappa}{2^{n}} \epsilon^{\lambda \mu \nu \mu_{1} \nu_{1} \ldots \mu_{n-1} \nu_{n-1}} \epsilon_{A B C D A_{1} B_{1} \ldots A_{n-1} B_{n-1}} \\
& \times F_{\mu_{1} \nu_{1}}^{A_{1} B_{1}} \ldots F_{\mu_{n-1} \nu_{n-1}}^{A_{n-1} B_{n-1}} \\
& \times\left\{2 n \delta_{\mu}^{\alpha} \delta A_{\nu}^{A B} £_{\Xi} A_{\lambda}^{C D}+F_{\mu \nu}^{A B}\left(2 \xi^{[\alpha} \delta_{\lambda}^{\gamma]} \delta A_{\gamma}^{C D}+\delta_{\lambda}^{\alpha} \delta \Xi_{(V)}^{C D}\right)\right\}
\end{aligned}
$$

Inserting (42) into (44) and integrating by parts the term containing the covariant derivative we obtain (second step):

$$
\mathcal{F}^{\alpha}\left(L^{\prime}\left(L_{1}, \Xi\right), X\right)=\tilde{\mathcal{F}}^{\alpha}\left(L^{\prime}\left(L_{1}, \Xi\right), X\right)+d_{\beta} \mathcal{U}^{\alpha \beta}\left(L^{\prime}\left(L_{1}, \Xi\right), X\right)
$$

where

$$
\tilde{\mathcal{F}}^{\alpha}\left(L^{\prime}\left(L_{1}, \Xi\right), X\right)=\delta\left\{-\frac{\kappa}{2^{n}} \epsilon^{\alpha \mu_{1} \nu_{1} \ldots \mu_{n} \nu_{n}} \epsilon_{A B A_{1} B_{1} \ldots A_{n} B_{n}} F_{\mu_{1} \nu_{1}}^{A_{1} B_{1}} \ldots F_{\mu_{n} \nu_{n}}^{A_{n} B_{n}} \Xi_{(V)}^{A B}\right\}
$$

\footnotetext{
${ }^{6}$ In the application $[23,26]$ of the formalism to stardard gravity we found that the BrownYork quasilocal energy [12] can be obtained when $\xi=\partial_{t}$ is the vector generating an ADM foliation of spacetime and $X$ corresponds to Dirichlet boundary conditions of the induced boundary three-metric.
} 
vanishes on-shell, while

$$
\begin{aligned}
\mathcal{U}^{\alpha \beta}\left(L^{\prime}\left(L_{1}, \Xi\right), X\right)= & \frac{n \kappa}{2^{n-1}} \epsilon^{\alpha \beta \rho \mu_{1} \nu_{1} \ldots \mu_{n-1} \nu_{n-1}} \epsilon_{A B C D A_{1} B_{1} \ldots A_{n-1} B_{n-1}} \\
& \times F_{\mu_{1} \nu_{1}}^{A_{1} B_{1}} \ldots F_{\mu_{n-1} \nu_{n-1}}^{A_{n-1} B_{n-1}} \delta A_{\rho}^{A B} \Xi_{(V)}^{C D}
\end{aligned}
$$

are the coefficients of the covariant potential $(2 n-1)$-form $\mathcal{U}=\frac{1}{2} \mathcal{U}^{\alpha \beta} d s_{\alpha \beta}$ with $\left.\left.d s_{\alpha \beta}=i_{\beta}\right\rfloor i_{\alpha}\right\rfloor d s$ (an expression similar to (47) was also obtained in [46] through a different technique). We remark that the integration of the potential gives rise, through expression (40), to the variation of the charge associated with the infinitesimal symmetry $\Xi$. Notice that the potential can be rewritten in a more compact form as follows ${ }^{7}$ :

$$
\begin{aligned}
\mathcal{U}\left(L^{\prime}\left(L_{1}, \Xi\right), X\right)= & n \kappa \epsilon_{A B C D A_{1} B_{1} \ldots A_{n-1} B_{n-1}} \\
& \times F^{A_{1} B_{1}} \wedge \ldots \wedge F^{A_{n-1} B_{n-1}} \wedge \delta A^{A B} \Xi_{(V)}^{C D}
\end{aligned}
$$

Since the potential has been obtained directly from the equations of motion and the equations of motion (6) are equivalent to field equations (14) we can immediately obtain the potential $\mathcal{U}\left(L^{\prime}\left(L_{2}, \Xi\right), X\right)$ relative to the latter equations. According to the schema (28) it is given by:

$$
\begin{aligned}
& \mathcal{U}\left(L^{\prime}\left(L_{2}, \Xi\right), X\right)=\frac{n \kappa}{l} \epsilon_{a b c a_{1} b_{1} \ldots a_{n-1} b_{n-1}} \\
& \times\left\{\hat{R}^{a_{1} b_{1}} \wedge \ldots \wedge \hat{R}^{a_{n-1} b_{n-1}} \wedge\left(\Xi_{(V)}^{a b} \delta e^{c}+\Xi_{(V)}^{c} \delta \omega^{a b}\right)\right. \\
& \left.+\hat{R}^{a_{2} b_{2}} \wedge \ldots \hat{R}^{a_{n-1} b_{n-1}} \wedge T^{c} \wedge \delta \omega^{a b} \Xi_{(V)}^{a_{1} b_{1}}\right\}
\end{aligned}
$$

with

$$
\Xi_{(V)}^{a}=e_{\mu}^{a} \xi^{\mu}, \quad \Xi_{(V)}^{a b}=\Xi^{a b}+\omega_{\mu}^{a b} \xi^{\mu}
$$

The result (50) can be easily inferred by inserting the splittings (7) and (8) into (49) and recalling the prescription $\Xi^{a}=0$ for the generators of symmetries. Alternatively, expression (50) can be obtained, in a clearly more involved way, from the variational Lagrangian

$$
\begin{aligned}
\left.L^{\prime}\left(L_{2}, \Xi\right), X\right)= & -(\kappa / l) \epsilon_{a a_{1} b_{1} \ldots a_{n} b_{n}} \hat{R}^{a_{1} b_{1}} \wedge \ldots \wedge \hat{R}^{a_{n} b_{n}} \wedge £_{\Xi} e^{a} \\
& -(n \kappa / l) \epsilon_{a a_{1} b_{1} \ldots a_{n} b_{n}} T^{a} \wedge \hat{R}^{a_{1} b_{1}} \wedge \ldots \wedge \hat{R}^{a_{n-1} b_{n-1}} \wedge £_{\Xi \omega^{a_{n} b_{n}}}
\end{aligned}
$$

(obtained from the contraction of the equations (14) with the Lie derivatives of fields with respect to the vector field (27)) via the two-step procedure outlined above.

Let us now turn the attention to the Lovelock-Chern-Simons theory described through the field equations $\frac{\delta L_{3}}{\delta g_{\lambda \rho}}=\frac{\partial L_{3}}{\partial g_{\lambda \rho}}$ ensuing from the Lagrangian

\footnotetext{
${ }^{7}$ According to our notation

$$
d x^{\mu_{1}} \wedge d x^{\nu_{1}} \wedge \ldots \wedge d x^{\mu_{n-1}} \wedge d x^{\nu_{n-1}} \wedge d x^{\rho}=\frac{1}{2} \epsilon^{\alpha \beta \rho \mu_{1} \ldots \nu_{n-1}} d s_{\alpha \beta}
$$
}


(18). ${ }^{8}$ The two-step calculation of the conserved quantities starting from the variational Lagrangian $L^{\prime}\left(L_{3}, \xi\right)=-\frac{\partial L_{3}}{\partial g_{\lambda \rho}} £_{\xi} g_{\lambda \rho}$ is obviously a rather cumbersome task. Nevertheless we have outlined in the introduction that conserved quantities can be calculated directly from (50) if we set $T=0$ and if we are able to select a suitable covariant lift of the spacetime vector field $\xi$ up to a vector field $\Xi$ into the configuration bundle $Y$ of the Lagrangian $L_{2}$. Namely if we are able to select a suitable rule $\Xi^{a b}=\Phi^{a b}\left(\xi^{\mu}, d_{\nu} \xi^{\mu}, \ldots\right)$ which determines the coefficients of $\Xi$ in terms of the components of $\xi$ and, obviously, in terms of the dynamical fields; see diagram (28). As already explained the prescription which allows to rule out the sought-for lift is the requirement that the variational Lagrangian $L^{\prime}\left(L_{2}, \Xi\right)$ and the variational Lagrangian $L^{\prime}\left(L_{3}, \xi\right)$ are equivalent (so that conserved quantities calculated from them are equivalent, too).

According to $[3,19,23,27,28]$ the generalized Kosmann lift $\Xi=K(\xi)$, obtained by the rule

$$
{ }^{K} \xi^{a b}={ }^{K} \xi^{[a b]}=e_{\alpha}^{[a} e^{b] \lambda} d_{\lambda} \xi^{\alpha}-\xi^{\gamma} e_{\mu}^{[a} d_{\gamma} e^{b] \mu}
$$

is the only one which restores the equality between the variational Lagrangians. This property is easily proved. Indeed, with the expression (53) we have

$$
£_{K(\xi)} e_{\mu}^{a}=\frac{1}{2} e^{a \nu} £_{\xi} g_{\mu \nu}
$$

so that

$$
\begin{aligned}
\left.L^{\prime}\left(L_{2}, K(\xi)\right)\right|_{T=0} & =-\frac{\delta L_{2}}{\delta e_{\mu}^{a}} £_{K(\xi)} e_{\mu}^{a}=-\frac{\partial L_{2}}{\partial e_{\mu}^{a}} £_{K(\xi)} e_{\mu}^{a} \\
& =-\frac{1}{2} \frac{\partial L_{2}}{\partial e_{\mu}^{a}} e^{a \nu} £_{\xi} g_{\mu \nu}=-\frac{\partial L_{3}}{\partial g_{\mu \nu}} £_{\xi} g_{\mu \nu} \\
& =-\frac{\delta L_{3}}{\delta g_{\mu \nu}} £_{\xi} g_{\mu \nu}=L^{\prime}\left(L_{3}, \xi\right)
\end{aligned}
$$

where we have taken into account that, if $T=0$, then $\left.L_{2}\left(e, j^{1} \omega\right)\right|_{T=0}=L_{3}(g(e))$ and $\frac{1}{2} \frac{\partial L_{2}}{\partial e_{\mu}^{a}} e^{a \nu}=\frac{\partial L_{3}}{\partial g_{\mu \nu}}$.

We also stress that the lift (53) is globally well defined. Indeed, the Kosmann lift $K(\xi)$ of a spacetime vector field $\xi$ transforms tensorially (as it can be inferred through a direct inspection) and can be therefore globally defined. Namely, all local expressions (53) can be patched together to define a unique global vector field; see [19, 27, 28].

It follows that the potential for the Lovelock-Chern-Simons theory can be obtained directly from (50) by setting $T=0$ and taking into account the pre-

\footnotetext{
${ }^{8}$ We remark that field equations are simply obtained by varying $L_{3}$ with respect to the metric alone since the variation of the curvature terms yields a total derivative. This is the reason why field equations are of second order only; [39, 40].
} 
scription (53), i.e.:

$$
\left\{\begin{aligned}
\mathcal{U}\left(L^{\prime}\left(L_{3}, \xi\right), X\right)= & \frac{n \kappa}{l} \epsilon_{a b c a_{1} b_{1} \ldots a_{n-1} b_{n-1}}\left({ }^{K} \xi_{(V)}^{a b} \delta e^{c}+{ }^{K} \xi_{(V)}^{c} \delta \omega^{a b}\right) \\
& \wedge \hat{R}^{a_{1} b_{1}} \wedge \ldots \wedge \hat{R}^{a_{n-1} b_{n-1}} \\
K_{\xi_{(V)}^{a}}^{a}=e_{\mu}^{a} \xi^{\mu}, \quad & K_{\xi_{(V)}^{a b}}^{a b}=e_{\alpha}^{[a} e^{b] \beta} \nabla_{\beta} \xi^{\alpha}
\end{aligned}\right.
$$

According to (40) the variation of conserved quantity (and therefore the variation of energy) is then obtained from the above expression after a suitable integration on a spacetime region.

Let us remark that it is also possible to separate into (55) the contributions ensuing from each Lagrangian $\overline{\mathcal{L}}_{p}$ of (18). Indeed, if we take the definition (15) into account expression (55) can be conveniently rewritten as:

$$
\mathcal{U}\left(L^{\prime}\left(L_{3}, \xi\right), X\right)=\kappa \sum_{p=0}^{n} \bar{c}_{p} \mathcal{U}_{p}
$$

where

$$
\begin{aligned}
\mathcal{U}_{p}= & \frac{p}{(2 n-2 p) !} \epsilon_{a b c a_{1} b_{1} \ldots a_{n-1} b_{n-1}}\left\{\left(e_{\alpha}^{a} e^{b \beta} \nabla_{\beta} \xi^{\alpha}\right) \delta e^{c}+\left(e_{\mu}^{c} \xi^{\mu}\right) \delta \omega^{a b}\right\} \\
& \wedge R^{a_{1} b_{1}} \wedge \ldots \wedge R^{a_{p-1} b_{p-1}} \wedge e^{a_{p}} \wedge e^{b_{p}} \wedge \ldots \wedge e^{a_{n-1}} \wedge e^{b_{n-1}}
\end{aligned}
$$

and $\bar{c}_{p}$ are given by (19). A direct comparison between (56) and (18) leads to our master formula

$$
\mathcal{U}\left(\overline{\mathcal{L}}_{p}\right)=\mathcal{U}_{p}
$$

for the covariant potential relative to the term $\overline{\mathcal{L}}_{p}$. Therefore we are now able to calculate the potential for a generic Lovelock Lagrangian

$$
L_{L o v}=\sum_{p=0}^{n} \alpha_{p} \overline{\mathcal{L}}_{p}\left(j^{2} g\right)
$$

with completely arbitrary constant coefficients $\alpha_{p}$. It turns out to be:

$$
\mathcal{U}_{\text {Lov }}=\sum_{p=0}^{n} \alpha_{p} \mathcal{U}_{p}
$$

In particular $\overline{\mathcal{L}}_{0}=\sqrt{g}$ gives no contribution to the potential, while the contribution ensuing from the Hilbert term $\overline{\mathcal{L}}_{1}=\sqrt{g} R$ is:

$$
\mathcal{U}_{1}^{\alpha \beta}=\sqrt{g} \delta_{\mu \nu \sigma}^{\alpha \beta \rho}\left(\nabla^{\nu} \xi^{\mu} e_{c}^{\sigma} \delta e_{\rho}^{c}+\xi^{\sigma} e_{a}^{\mu} e_{b}^{\nu} \delta \omega_{\rho}^{a b}\right)
$$

Formula (61) reproduces exactly the formula for the variation of conserved quantities found in $[10,12,26,37]$ for General Relativity. This property was proven explicitly in [3] when the spacetime dimension is three; but the same calculation can be carried on, step by step, in any higher spacetime dimension. 
We also point out the remarkable simple expression (57) and (60) for the potential in Lovelock gravity. In the sequel we shall test its viability, i.e. we shall analyse whether formula (60) allows to reproduce physically expected quantities in specific applications.

Remark 2.1 Let us consider again formula (49). It is straightforward to verify that, on-shell, the potential satisfies:

$$
d \mathcal{U}\left(L^{\prime}\left(L_{1}, \Xi\right), X\right)=\omega\left(L_{1}, X, £_{\Xi} A\right)
$$

where the $(D-1)$-form:

$$
\begin{aligned}
\omega\left(L_{1}, X, £_{\Xi} A\right)= & n \kappa \epsilon_{A B C D A_{1} B_{1} \ldots A_{n-1} B_{n-1}} \\
& \times F^{A_{1} B_{1}} \wedge \ldots \wedge F^{A_{n-1} B_{n-1}} \wedge £_{\Xi} A^{A B} \wedge \delta_{X} A^{C D}
\end{aligned}
$$

is the symplectic current relative to $L_{1}$; see [46]. This remarkable property ensures that we can make the identification

$$
\mathcal{U}\left(L^{\prime}\left(L_{1}, \Xi\right), X\right)=\delta_{X} \mathcal{V}\left(L_{1}, \Xi\right)-B\left(L_{1}, \Xi, X\right)
$$

where $\mathcal{V}\left(L_{1}, \Xi\right)$ is the Noether superpotential, while $B\left(L_{1}, \Xi, X\right)$ is the so-called Regge-Teitelboim covariant correction term, namely the term which has to be added to the variation of the Hamiltonian to have a well-posed variational problem (or, in other words, it is the factor which exactly cancels the boundary term coming from the variation of the Hamiltonian; see [22, 34, 36, 44]). This property, a posteriori, endows the definition we suggested for the variation of the energy, i.e:

$$
\delta_{X} E_{\Sigma}(\Xi)=\int_{\partial \Sigma} \mathcal{U}\left(L^{\prime}\left(L_{1}, \Xi\right), X\right) \simeq \int_{\Sigma} \omega\left(L_{1}, X, £_{\Xi} A\right)
$$

with a well-defined formal meaning since it is coherent with a (Hamiltonian) symplectic description of field theories.

Moreover, if $\Xi$ is a Killing vector for the solution, i.e. $£_{\Xi} A=0$, the symplectic form $\omega$ is vanishing, the potential $\mathcal{U}$ becomes a closed form and formula (65) is the conservation law for energy; see [3, 36, 37].

Inserting (7) and (8) into (62) and setting $T=0$ and $\Xi=K(\xi)$, we easily obtain the conservation law for Lovelock-Chern-Simons theory:

$$
d \mathcal{U}\left(L^{\prime}\left(L_{3}, K(\xi)\right), X\right)=\omega\left(L_{3}, X, £_{K(\xi)} \omega, £_{K(\xi)} e\right)
$$

where the symplectic current [47] is now given by:

$$
\begin{aligned}
\omega\left(L_{3}, X, £_{\Xi} \omega, £_{\Xi} e\right)= & \frac{n \kappa}{l} \epsilon_{a b c a_{1} b_{1} \ldots a_{n-1} b_{n-1}} \hat{R}^{a_{1} b_{1}} \wedge \ldots \wedge \hat{R}^{a_{n-1} b_{n-1}} \\
& \wedge\left(£_{K(\xi)} e^{a} \wedge \delta \omega^{b c}-\delta e^{a} \wedge £_{K(\xi)} \omega^{b c}\right)
\end{aligned}
$$


and is vanishing whenever $K(\xi)$ is a Killing vector for the dynamical fields $\left(e^{a}, \omega^{b c}\right) .^{9}$

Remark 2.2 The electromagnetic field can be included into the theory simply by minimal coupling the electromagnetic field to the Lovelock-Chern-Simons Lagrangian (18), i.e.

$$
L_{L o v}^{e m}=L_{3}\left(j^{2} g\right)+L_{e m}(g, F)
$$

where $L_{e m}(g, F)$ is the Maxwell Lagrangian in $(2 n+1)$ dimensions:

$$
L_{e m}(g, F)=-\frac{1}{4 \Omega_{D-2}} \sqrt{g} F_{\mu \nu} F_{\alpha \beta} g^{\mu \alpha} g^{\nu \beta}
$$

The potential relative to the pure electromagnetic part can be calculated, through the two-step procedure outlined in section 2 , starting from the variational Lagrangian

$$
L^{\prime}\left(L_{e m},(\xi, \chi)\right)=-\frac{\delta L_{e m}}{\delta A_{\mu}} £_{(\xi, \chi)} A_{\mu}-\frac{\delta L_{e m}}{\delta g_{\mu \nu}} £_{\xi} g_{\mu \nu}
$$

where

$$
\left\{\begin{array}{l}
£_{(\xi, \chi)} A_{\mu}=\xi^{\rho} d_{\rho} A_{\mu}+d_{\mu} \xi^{\rho} A_{\rho}+d_{\mu} \chi \\
£_{\xi} g_{\mu \nu}=\nabla_{\mu} \xi_{\nu}+\nabla_{\nu} \xi_{\mu}
\end{array}\right.
$$

We shall here skip the details of the calculation since they are just a mere and straightforward application of the formalism. The result turns out to be:

$$
\begin{aligned}
\mathcal{U}^{\alpha \beta}\left(L^{\prime}\left(L_{e m},(\xi, \chi)\right), X\right)= & -\frac{1}{\Omega_{D-2}} \delta\left(\sqrt{g} F^{\alpha \beta}\right)\left(\chi+A_{\rho} \xi^{\rho}\right) \\
& -\frac{\sqrt{g}}{\Omega_{D-2}}\left(F^{\alpha \beta} \xi^{\rho}+2 \xi^{[\alpha} F^{\beta] \rho}\right) \delta A_{\rho}
\end{aligned}
$$

It then follows the the potential for the Lagrangian (69) is obtained from (55) and (73), i.e.

$$
\mathcal{U}^{\alpha \beta}\left(L^{\prime}\left(L_{L o v}^{e m},(\xi, \chi)\right), X\right)=\mathcal{U}^{\alpha \beta}\left(L^{\prime}\left(L_{3}, \xi\right), X\right)+\mathcal{U}^{\alpha \beta}\left(L^{\prime}\left(L_{e m},(\xi, \chi)\right), X\right)
$$

\section{The $D=5$ Spherically Symmetric Charged So- lution}

To check the reliability of the formula (74) we test it on an exact solution and we show that it allows to recover the correct expression for the first law of black

\footnotetext{
${ }^{9}$ We remark that the Lie derivative of the vielbein with respect to the Kosmann lift is given by (54), while the Lie derivative of the spin connection turns out to be [23]:

$$
£_{K(\xi)} \omega_{\mu}^{a b}=e_{\lambda}^{[a} e^{b] \gamma} £_{\xi} \gamma^{\lambda} \gamma \mu
$$

where $\gamma^{\lambda} \gamma \mu$ are the Christoffel symbols of the metric $g_{\mu \nu}=\eta_{a b} e_{\mu}^{a} e_{\nu}^{b}$.
} 
holes mechanics when stationary black holes solutions for the 5-dimensional Lovelock-Maxwell gravity are considered $[6,7,11,15,16]$.

Let us consider a five dimensional solution of the field equations ensuing from the $D=5$ Lagrangian (69). The gravitational contribution to the total gravitation-electromagnetic potential (74) can be read out from formula (56) with $n=2$. It is given by:

$$
\mathcal{U}^{\alpha \beta}\left(L^{\prime}\left(L_{3}, \xi\right), X\right)=\mathcal{U}^{\alpha \beta}\left(L^{\prime}\left(L_{H}, \xi\right), X\right)+\mathcal{U}^{\alpha \beta}\left(L^{\prime}\left(L_{G B}, \xi\right), X\right)
$$

where

$$
\mathcal{U}^{\alpha \beta}\left(L^{\prime}\left(L_{H}, \xi\right), X\right)=\frac{4 \kappa}{l^{3}} \sqrt{g} \delta_{\mu \nu \sigma}^{\alpha \beta \rho}\left(\nabla^{\nu} \xi^{\mu} e_{c}^{\sigma} \delta e_{\rho}^{c}+\xi^{\sigma} e_{a}^{\mu} e_{b}^{\nu} \delta \omega_{\rho}^{a b}\right)
$$

and:

$$
\mathcal{U}^{\alpha \beta}\left(L^{\prime}\left(L_{G B}, \xi\right), X\right)=\frac{1}{12 \pi^{2}} \epsilon^{\alpha \beta \rho \sigma \nu} \epsilon_{a b c d e} R_{\rho \sigma}^{a b}\left(e_{\mu}^{e} \xi^{\mu} \delta \omega_{\nu}^{c d}+e_{\mu}^{d} e^{e \gamma} \nabla_{\gamma} \xi^{\mu} \delta e_{\nu}^{c}\right)
$$

are the contributions ensuing, respectively, from the Hilbert Lagrangian $\overline{\mathcal{L}}_{1}$ and the Gauss-Bonnet Lagrangian $\overline{\mathcal{L}}_{2}$. The electromagnetic contribution to the total potential (74) is instead given by formula (73):

$$
\begin{aligned}
\mathcal{U}^{\alpha \beta}\left(L^{\prime}\left(L_{e m},(\xi, \chi)\right), X\right)= & -\frac{1}{2 \pi^{2}} \delta\left(\sqrt{g} F^{\alpha \beta}\right)\left(\chi+A_{\rho} \xi^{\rho}\right) \\
& -\frac{\sqrt{g}}{2 \pi^{2}}\left(F^{\alpha \beta} \xi^{\rho}+2 \xi^{[\alpha} F^{\beta] \rho}\right) \delta A_{\rho}
\end{aligned}
$$

We remark that the area $\Omega_{3}$ of the three dimensional unit sphere is $12 \pi^{2}$ so that, from (5), we infer that the coupling constant $\kappa$ turns out to be $\kappa=l / 12 \pi^{2}$.

The spherically symmetric charged black hole solution is; see [6]:

$$
\left\{\begin{array}{l}
d s^{2}=-f^{2}(r) d t^{2}+\frac{1}{f^{2}(r)} d r^{2}+r^{2} d \Omega_{3} \\
f^{2}(r)=1+\frac{r^{2}}{l^{2}}-\sqrt{M+1-\frac{Q^{2}}{4 r^{2}}} \\
A=\varphi d t, \quad \varphi=\frac{Q}{2 r^{2}}
\end{array}\right.
$$

where $d \Omega_{3}$ is the standard metric on the three-dimensional unit sphere. The event horizon is located at $r=r_{+}$, where $r_{+}$is the positive solution of $f^{2}\left(r_{+}\right)=$ 0 ; see [6]. If we now consider the vector field $(\xi, \chi)=\left(\partial_{t}, \chi_{0}\right)$, with $\chi_{0}=$ constant, and we integrate expression (74) on a three-sphere $S$ of constant $r$ and $t$ (with $r>r_{+}$) we obtain

$$
\delta_{X} Q_{3}(S, \xi)=\delta M-\frac{Q \delta Q}{2 r^{2}}
$$

from the gravitational part (75); and

$$
\delta_{X} Q_{e m}\left(S, \xi, \chi_{0}\right)=\frac{Q \delta Q}{2 r^{2}}+\chi_{0} \delta Q
$$


from the electromagnetic part (78). The variation of the total charge is therefore obtained by their sum:

$$
\delta_{X} Q_{L o v}^{e m}(S, \xi, \chi)=\delta M+\chi_{0} \delta Q
$$

and reproduces the espected physical values. We remark that even though (80) and (81) depend explicitly on the radius of the sphere, their sum (82) does not depend on it. This property follows from the consideration we made in Remark 2.1. Indeed from $(72)$ we can easily infer that the vector field $\left(\xi, \chi_{0}\right)$ is a Killing vector field for the solution (79) and consequently the potential (74) is a closed three-form. Therefore:

$$
\delta_{X} Q_{L o v}^{e m}\left(S, \xi, \chi_{0}\right)=\delta_{X} Q_{L o v}^{e m}\left(S^{\prime}, \xi, \chi_{0}\right)
$$

whenever $S$ and $S^{\prime}$ are homologous surfaces.

In particular, we can identify $\chi_{0}$ with (minus) the electric potential on the horizon $\chi_{0}=-\varphi\left(r_{+}\right)=-\frac{Q}{2 r_{+}^{2}}$ (the thermodynamical parameter conjugated to the charge). In this case formula (82) becomes the first law of black hole thermodynamics:

$$
\delta M-\varphi\left(r_{+}\right) \delta Q=T \delta S
$$

where:

$$
T=\frac{1}{4 \pi}\left[\frac{2 r_{+}}{l^{2}}-\frac{Q^{2} l^{2}}{4 r_{+}^{3}\left(l^{2}+r_{+}^{2}\right)}\right]
$$

is the temperature $T=\left.\frac{1}{4 \pi} \frac{d f^{2}(r)}{d r}\right|_{r=r_{+}}$of the black hole, while

$$
S=8 \pi\left[r_{+}+\frac{r_{+}^{3}}{3 l^{2}}\right]
$$

is its entropy; see [6]. In obtaining this result, in the right hand side of (84) we have taken into account that the mass and the charge of the solution are related to the radius of the horizon $r_{+}$via the relation $f^{2}\left(r_{+}\right)=0$.

\section{The Entropy in Lovelock Gravity}

As it is manifest from (86) the entropy for the spherically symmetric charged solution differs from the area law of Einstein gravity. This is a well-known result. It was indeed demonstrated in [35] that in Lovelock gravity each higher derivative term gives a contribution to the entropy in its own. We shall now analyse to what extent formulae (57) and (60) are in agreement with the results already existing in literature.

Let us consider the Lovelock Lagrangian (59) and let us consider a stationary black hole solution of its field equations, admitting a Killing horizon $\mathcal{H}$ and a bifurcation surface $H$; see $[33,35,48,50]$. Let us denote by $\xi$ the unique Killing 
vector field which is null on the horizon and vanishes on $H$, i.e. the vector field which describes the evolution of a system of ROTORS (co-rotating observers; see [49]). We remark that on the bifurcation surface it holds true that:

$$
\nabla_{\mu} \xi_{\nu}=-2 \kappa_{H} \beta_{\mu \nu}
$$

where $\kappa_{H}$ is the surface gravity and $\beta_{\mu \nu}$ denotes the binormal to $H$.

Let us then consider a spacelike hypersurface $\Sigma$ which extends from spatial infinity to the horizon and intersects the horizon in the bifurcation surface. Since $\xi$ is transverse to $D$ the variation of energy is identified with the the integral $\int_{S} \mathcal{U}(\xi, X)$ of the potential $\mathcal{U}$ on a $(2 n-1)$-dimensional surface $S$ enclosing the singularity. But the potential is a closed form since $\xi$ is a Killing vector (see Remark 2.1). Therefore the integral $\int_{S} \mathcal{U}(\xi, X)$ is equal to $\int_{S^{\prime}} \mathcal{U}(\xi, X)$ for any surface $S^{\prime}$ homologous to $S$. In particular we can identify $S^{\prime}$ with the bifurcation surface $H$ and calculate the variation of the energy on it:

$$
\delta E(\xi)=\int_{H} \frac{1}{2} \mathcal{U}_{L o v}^{\alpha \beta} d s_{\alpha \beta}=\sum_{p=0}^{n} \alpha_{p} \int_{H} \frac{1}{2} \mathcal{U}_{p}^{\alpha \beta} d s_{\alpha \beta}
$$

where the potentials $\mathcal{U}_{p}^{\alpha \beta}$ are given by (57). As the vector $\xi$ vanishes on $H$ only terms containing derivatives of $\xi$ survive into (88), i.e.

$$
\begin{aligned}
\left.\mathcal{U}_{p}^{\alpha \beta}\right|_{H}= & \frac{p}{(2 n-2 p) ! 2^{p-1}} \epsilon^{\alpha \beta \rho \mu_{1} \nu_{1} \ldots \mu_{n-1} \nu_{n-1}} \epsilon_{a b c a_{1} b_{1} \ldots a_{n-1} b_{n-1}} \\
& \times\left\{R_{\mu_{1} \nu_{1}}^{a_{1} b_{1}} \ldots R_{\mu_{p-1} \nu_{p-1}}^{a_{p-1} b_{p-1}} e_{\mu_{p}}^{a_{p}} e_{\nu_{p}}^{b_{p}} \ldots e_{\mu_{n-1}}^{a_{n-1}} e_{\nu_{n-1}}^{b_{n-1}}\right\} \\
& \times e_{\gamma}^{a} e^{b \sigma} \nabla_{\sigma} \xi^{\gamma} \delta e_{\rho}^{c}
\end{aligned}
$$

Taking into account the property (87) together with the integration rule

$$
\int_{S} \frac{1}{2} \sqrt{g} \mathcal{V}^{[\alpha \beta]} d s_{\alpha \beta}=\int_{S} \sqrt{\tilde{g}} d^{D-2} x \mathcal{V}^{[\alpha \beta]} \beta_{\alpha \beta}
$$

(which holds true for any $(D-2)$-form $\mathcal{V}=\frac{1}{2} \sqrt{g} \mathcal{V}^{[\alpha \beta]} d s_{\alpha \beta}$ and any $(D-2)$ region $S$ with induced metric $\tilde{g}$ and binormal $\beta_{\alpha \beta}=n_{[\alpha} u_{\beta]}$, where $n_{\alpha}$ and $u_{\beta}$ are the outward pointing unit normals to $S$ ) we obtain:

$$
\begin{aligned}
& \int_{H} \frac{1}{2} \mathcal{U}_{p}^{\alpha \beta} d s_{\alpha \beta}=\frac{2 p \kappa_{H}}{(2 n-2 p) ! 2^{p-1}} \int_{H} \sqrt{\tilde{g}} d^{D-2} x \\
& \times \tilde{\epsilon}^{\rho \mu_{1} \nu_{1} \ldots \mu_{n-1} \nu_{n-1}} \tilde{\epsilon}_{\lambda \alpha_{1} \beta_{1} \ldots \alpha_{p-1} \beta_{p-1} \mu_{p} \nu_{p} \ldots \nu_{n-1}} R_{\mu_{1} \nu_{1}}^{\alpha_{1} \beta_{1}} \ldots R_{\mu_{p-1} \nu_{p-1}}^{\alpha_{p-1} \beta_{p-1}} e_{c}^{\lambda} \delta e_{\rho}^{c}
\end{aligned}
$$

where $\tilde{\epsilon}^{\mu_{1} \ldots \mu_{2 n-1}}=\epsilon^{\mu \nu \mu_{1} \ldots \mu_{2 n-1}} \beta_{\mu \nu}$ is the $(2 n-1)$-dimensional Levi-Civita totally skew-symmetric tensor density (from now on a "tilde" over a quantity will always denote the projection of the quantity itself onto the surface $H$ ). Notice that in the above formula the Riemann tensors are fully projected onto the surface $H$. Moreover, since $H$ is a bifurcation surface, its extrinsic curvature vanishes and (via the Gauss-Codazzi equations [41, 50]) we can identify the 
projected Riemann tensor with the $(2 n-1)$-dimensional Riemann tensor $\tilde{R}$ built out of the induced $(2 n-1)$-metric $\tilde{g}_{\mu \nu}$, see [35]. Contracting the product of the Levi-Civita tensor densities in (91) and rewriting them as a product of $(2 n-1)$-dimensional Kronecker deltas $\tilde{\delta}_{\nu}^{\mu}$ we finally obtain:

$\delta E_{p}(\xi):=T \delta S_{p}=\frac{p \kappa_{H}}{2^{p-2}} \int_{H} \sqrt{\tilde{g}} d^{D-2} x \tilde{\delta}_{\lambda \alpha_{1} \beta_{1} \ldots \alpha_{p-1} \beta_{p-1}}^{\rho \mu_{1} \nu_{1} \ldots \mu_{p-1} \nu_{p-1}} \tilde{R}_{\mu_{1} \nu_{1}}^{\alpha_{1} \beta_{1}} \ldots \tilde{R}_{\mu_{p-1} \nu_{p-1}}^{\alpha_{p-1} \beta_{p-1}} \tilde{e}_{c}^{\lambda} \delta \tilde{e}_{\rho}^{c}$

where the temperature $T$ is given by $T=\frac{\kappa_{H}}{2 \pi}$. As recognized in [35] the above expression can be formally integrated since:

$$
\sqrt{\tilde{g}} \tilde{\delta}_{\lambda \alpha_{1} \beta_{1} \ldots \alpha_{p-1} \beta_{p-1}}^{\rho \mu_{1} \nu_{1} \ldots \mu_{p-1} \nu_{p-1}} \tilde{R}_{\mu_{1} \nu_{1}}^{\alpha_{1} \beta_{1}} \ldots \tilde{R}_{\mu_{p-1} \nu_{p-1}}^{\alpha_{p-1} \beta_{p-1}} \tilde{e}_{c}^{\lambda} \delta \tilde{e}_{\rho}^{c}=2^{p-1} \delta \overline{\mathcal{L}}_{(p-1)}
$$

Extracting the temperature from (92) we thence obtain:

$$
S_{p}=4 \pi p \int_{H} \overline{\mathcal{L}}_{(p-1)}(\tilde{g})
$$

namely, the contribution to the entropy ensuing from the term $\overline{\mathcal{L}}_{p}(g)$ of the Lovelock Lagrangian is obtained from the Lagrangian $\overline{\mathcal{L}}_{(p-1)}(\tilde{g})$ defined with respect to the induced $(2 n-1)$-metric $\tilde{g}_{\mu \nu}$. Therefore (88) corresponds to the first law of black holes thermodynamics ${ }^{10}$ :

$$
\delta E(\xi)=T \delta S
$$

where

$$
S=4 \pi \sum_{p=1}^{n} p \alpha_{p} \int_{H} \overline{\mathcal{L}}_{(p-1)}(\tilde{g})
$$

This result agrees exactly with the result obtained by Wald within a Lagrangian approach [33] or by Jacobson-Myers within a Hamiltonian method [35].

We stress again that (88) is the integral of a closed form (since $\xi$ is a Killing vector) and therefore the integral on $H$ equals the integral on any other surface $S$ homologous to $H$ itself. Nevertheless on any other surface other than $H$ the formal calculation of $\delta E(\xi)=\int_{S} U(\xi, X)$ becomes rather more involved since also the terms linear in $\xi$ have to be retained and the simplifications related to the particular geometry of the bifurcation surface do not hold true any longer. Despite of this, even though the formal calculation above requires the presence of the bifurcation surface, on the contrary, as we did already remark in [20, 21], its existence become a unessential requirement in specific applications. In the charged spherical symmetric solution we have dealt with in section 3, e.g., the bifurcation surface is out of the domain of coordinates (and its presence would be revealed only in the maximal analytic extension with Kruskal-Szekeres-like

\footnotetext{
${ }^{10}$ We remark that, according to our choice of the vector $\xi$, the variation $\delta E$ of energy turns out to be $\delta E=\delta M-\Omega^{(i)} J_{(i)}$ where: $M$ is the mass of the black hole, $J_{(i)}$, with $i=1 \ldots n$, are the angular momenta associated with the commuting Killing vectors generating spatial rotations and $\Omega^{(i)}$ are the horizon angular velocities; see [16, 35].
} 
coordinates; [31, 33, 43, 48]). However the first law (84) of black hole mechanics has been established performing calculation on any surface enclosing the singularity. This noteworthy property, we stress again, follows from the cohomological properties the potential inherits in presence of a Killing vector.

\section{Acknowledgments}

We are grateful to A. Borowiec of the University of Wroclaw for having directed our attention to the Chern-Simons formulation of Lovelock gravity. We also thank L. Fatibene and M. Ferraris of the University of Torino for useful discussions and suggestions on the subject.

\section{References}

[1] A.Achucarro, P.K.Townsend, Phys.Lett. B 180 (1986), 89.

[2] G. Allemandi, M. Francaviglia, M. Raiteri: Class. Quant. Grav. 19 (2002), 2633-2655 (gr-qc/0110104).

[3] G. Allemandi, M. Francaviglia, M. Raiteri: Class. Quant. Grav. 20 (2003), 483-506 (gr-qc/0211098).

[4] S.C.Anco, R.S.Tung, J. Math. Phys 43 (2002), 3984;

S.C.Anco, R.S.Tung, J. Math. Phys 43 (2002), 5531.

[5] M. Bañados, C. Teitelboim, J. Zanelli: Phys. Rev. Lett. 69 (1992), 18491851 (hep-th/9204099);

M. Bañados, M. Henneaux, C. Teitelboim, J. Zanelli: Phys. Rev. D 48 (1993), 1506-1525 (gr-qc/9302012).

[6] M. Bañados, C. Teitelboim, J. Zanelli: Phys. Rev. D 49 (2) (1994), 975-986 $(\mathrm{gr}-\mathrm{qc} / 9307033)$.

[7] M. Bañados: Phys. Rev. D 65 (2002), (hep-th/0109031);

M. Bañados: Nucl. Phys. Proc. Suppl. 88 (2000), 17-26 (hep-th/9911150).

[8] G. Barnich, F. Brandt, Nucl.Phys. B633 (2002) 3 (hep-th/0111246).

[9] N.D. Birrel, P. C. W. Davies: Quantum fields in curved space, Cambridge University Press (1982).

[10] I. Booth: PhD Thesis from University of Waterloo (gr-qc/0008030);

I. Booth, R.B. Mann: Phys. Rev. D 59, 064021 (gr-qc/9810009);

I. Booth, R.B. Mann: Phys. Rev. D 124009 (1999) (gr-qc/9907072).

[11] D.G. Boulware, S. Deser: Phys. Rev. Lett. 55 (24) (1985), 2656-2660. 
[12] J. D. Brown, J. W. York, Phys. Rev. D 47 (4), (1993),1407;

J. D. Brown, J. W. York, Phys. Rev. D 47 (4) (1993), 1420.

[13] A. H. Chamseddine: Nucl. Phys. B 346 (1990), 213-234;

A. H. Chamseddine, J. Fröhlich: Comm. Math. Phys. 147 (1992), 549.

[14] C. M. Chen, J. M. Nester: Gravitation \& Cosmology 6, 257, (2000) (grqc/0001088).

[15] J. Crisostomo, R. Troncoso, J. Zanelli: Phys.Rev. D 62 (2000), 084013 (hep-th/0003271).

[16] M. H. Dehghani: Phys. Rev. D 67 (2003), 064017 (hep-th/0211191).

[17] S. Deser, B. Tekin: Phys. Rev. D 67 (2003), 084009 (hep-th/0212292).

[18] L. Fatibene and M. Francaviglia, Natural and gauge natural formalism for classical field theories: a geometric perspective including spinors and gauge fields, in Pubblication for Kluwer Academic Publishers.

[19] L. Fatibene, M. Ferraris, M. Francaviglia, M. Godina: Gen. Rel. Grav. 30 (1998), 1371-1389.

[20] L. Fatibene, M. Ferraris, M. Francaviglia, M. Raiteri: Annals of Phys. 275 (1999), 27.

[21] L. Fatibene, M. Ferraris, M. Francaviglia, M. Raiteri, Phys. Rev. D60, 124012 (1999);

L. Fatibene, M. Ferraris, M. Francaviglia, M. Raiteri, Phys. Rev. D60, 124013 (1999);

L. Fatibene, M. Ferraris, M. Francaviglia, M. Raiteri, Annals of Phys. 284, 197 (2000), gr-qc/9906114.

[22] M. Ferraris and M. Francaviglia: Atti Sem. Mat. Univ. Modena, 37 (1989), 61; M. Ferraris and M. Francaviglia: Gen. Rel. Grav., 22, (9) (1990), 965; M. Ferraris, M. Francaviglia, I. Sinicco: Il Nuovo Cimento, 107B, N. 11, (1992), 1303; M. Ferraris, M. Francaviglia: 7th Italian Conference on General Relativity and Gravitational Physics, Rapallo (Genoa), September 3-6, 1986.

[23] M. Ferraris, M. Francaviglia, M. Raiteri: Class. Quant. Grav. in press (grqc/0305047).

[24] M. Ferraris, M. Francaviglia, in: Mechanics, Analysis and Geometry: 200 Years after Lagrange, Editor: M. Francaviglia, Elsevier Science Publishers B.V., (Amsterdam, 1991) 451;

A. Trautman, in: Gravitation: An Introduction to Current Research, L. Witten ed. (Wiley, New York, 1962) 168;

A. Trautman, Commun. Math. Phys., 6, (1967), 248. 
[25] M. Ferraris, M. Francaviglia and O. Robutti, in: Geometrie et Physique, Proceedings of the Journees Relativistes 1985 (Marseille, 1985), 112 - 125; Y. Choquet-Bruhat, B. Coll, R. Kerner, A. Lichnerowicz eds. (Hermann, Paris, 1987).

[26] M. Francaviglia, M. Raiteri: Class. Quant. Grav. 19, (2002), 237-258 (grqc/0107074).

[27] M. Godina, P. Matteucci: in press for J. Geom. Phys. (math.DG/0201235);

P. Matteucci, gr-qc/0201079.

[28] M. Godina, P. Matteucci, J. A. Vickers: J. Geom. Phys. 39 (2001), 265-275.

[29] H.Goldschmidt, D.Spencer, J. Diff. Geom. 13 (1978) 455.

[30] M.B. Greens, J.H. Schwarz, E.Witten: Superstring Theory (Cambridge University Press, England 1987);

D. Lust, S. Theusen: Lectures on String Theory (Springer, Berlin, 1989); J.Polchinski: String Theory (Cambridge University Press, England 1998).

[31] S. W. Hawking, G.F.R. Ellis, The Large Scale Structure of Space-Time, (Cambridge University Press, Cambridge 1973).

[32] S. W. Hawking, C. J. Hunter, Class. Quant. Grav. 13, (1996) 2735;

S. W. Hawking, G. T. Horowitz, Class. Quant. Grav. 13, (1996) 1487.

[33] V. Iyer and R. Wald: Phys. Rev. D 50 (1994), 846 (gr-qc/9403028).

[34] V. Iyer, R. M. Wald: Phys.Rev. D 52 (1995), 4430-4439 (gr-qc/9503052).

[35] T.Jacobson, R. Myers: Phys. Rev. Lett. 70, 3684-3687 (1993) (hepth/9305016).

[36] B. Julia, S. Silva: Class. Quant. Grav. 15, (1998), 2173 - 2215 (grqc/9804029);

B. Julia, S. Silva: Class. Quant. Grav. 17 (2000), 4733 - 4744 (grqc/0005127).

[37] J. Kijowski: Gen. Rel. Grav. 29 (1997), 307.

[38] I. Kolàr̆, P.W. Michor, J. Slovàk: Natural Operations in Differential Geometry, Springer-Verlag (New York, 1993).

[39] C. Lanczos: Ann. Math. 39 (1938), 842.

[40] D. Lovelock: J. Math. Phys. 12 (1971), 498;

D. Lovelock: J. Math. Phys. 13 (1972), 874.

[41] C.W. Misner, K.S. Thorne, J.A. Wheeler, Gravitation (Freeman, San Francisco, 1973). 
[42] R. C. Myers: Phys. Rev. D 36 (1987), 392.

[43] I. Racz, R. M. Wald: (gr-qc/9507055).

[44] T. Regge, C. Teitelboim: Annals of Physics 88 (1974), 286.

[45] G. Sardanashvily: Generalized Hamiltonian Formalism for Field Theory, World Scientific (Singapore, 1995).

[46] S. Silva, Nucl. Phys. B 558, (1999), 391 (hep-th/9809109).

[47] C. Teitelboim, J. Zanelli: in Constraints Theory and Relativistic Dynam$i c s$, Proceedings of the Workshop held in Florence, Arcetri, Italy (World Scientific, Singapore 1987), 94.

[48] P. K. Townsend: Black Holes, DAMPT University of Cambridge.

[49] M. Visser: Phys. Rev. D 48 (1993), 5697-5705 (hep-th/9307194).

[50] R. M. Wald: General Relativity, University of Chicago Press (Chicago, 1984).

[51] E. Witten: Nucl. Phys. B 311 (1988), 96;

E. Witten: Nucl. Phys. B 323 (1989), 113. 